\title{
Axial acoustic field barrier for fluidic particle manipulation
}

\author{
Nan Li, ${ }^{1}$ Akshay Kale, ${ }^{1}$ and Adrian C. Stevenson ${ }^{1, a)}$ \\ Department of Chemical Engineering and Biotechnology, University of Cambridge, Philippa Fawcett Drive, \\ Cambridge, CB3 OAS, UK
}

(Dated: 18 January 2019)

An acoustic field barrier integrated within a flow tubing system to achieve high-throughput separation of particles in fluid is reported in this work. We investigate the axial acoustic field of a piezo-tube with an inside diameter $34 \mathrm{~mm}$, length $25 \mathrm{~mm}$ and operating frequency $1.15 \mathrm{MHz}$. Energy concentrates within the tube and leakage at the ends provides a sharp monotonic acoustic pressure field within a fluidic circuit. This process is not the conventional standing wave mechanism; instead the geometry produces a spatially stable filtering action without fouling. This powerful filtering action is confirmed theoretically via a COMSOL simulation and demonstrated experimentally by concentrating suspensions of $5 \mu \mathrm{m}$ proteoglycan tracer particles at a flow rate of $20 \mathrm{~mL} / \mathrm{min}$ : The corresponding acoustic contrast factor is 0.243 and trapping force is $11 \mathrm{pN}$. This tube geometry tackles the limitations of microfluidic standing wave based acoustic concentrators, namely complex extraction, low-throughput and distributed focus, by harnessing a stable monotonic field profile.

Keywords: acoustophoresis, acoustic, focussing, microparticle, trapping, piezoelectric, piezo

Acoustic pressure fields offer non-contact manipula- 53 tion opportunities, with levitation in air receiving atten- 54 tion recently with stable single beam levitation ${ }^{1}$, mid-air 55 trapping and control $^{2}$, single axis levitation ${ }^{3}$ and non- 56 spherical particles in artificial field profiles ${ }^{4}$. Levitation 57 of microparticles in liquids has also emerged within ad- 58 vanced healthcare, biotechnology research, and industrial 59 applications such as cell culturing ${ }^{5}$, early diagnosis of 60 diseases $^{6}$, biomass harvesting ${ }^{7}$ and food quality control $^{8}$. 61

These standing wave pressure fields have high particle concentration efficiency, rapid processing and maintenance of cell viability ${ }^{9}$. The primary advantage is access to physical forces that localise particles at an equilibrium ${ }^{62}$ point in a similar fashion as acoustic tweezers ${ }^{10}$. The most successful application is miniaturised lab-on-a-chip geometries ${ }^{9}$ with standing wave patterns in submillimetre ${ }^{63}$ sized channels ${ }^{11}$. Particle separation is well controlled for ${ }^{64}$ half-wavelength standing wave gaps with two monotonic ${ }^{65}$ pressure field profiles straddling a focussing point ${ }^{12}$. 66

Widening standing wave gaps to increase throughput ${ }^{67}$ produces polytonic regions(FIG.1.(a)) with distributed 68 focus. This loss of focus and potential de-tune indicates standing waves in larger structures have an Achilles' ${ }_{69}$ heel, i.e. particles dont move to a single equilibrium point. Whereas living organisms sticking together via acoustic pressure waves improves harvesting ${ }^{13,14}$ and ${ }^{70}$ throughput $^{15}$. Nevertheless filtration, centrifugation, ${ }^{71}$ flocculation, sedimentation ${ }^{16}$ remain attractive options. ${ }^{72}$

We present a piezo-tube method to create monotonic ${ }^{73}$ pressure profiles with single focussing action(FIG.1.(b)). ${ }^{74}$ It patches the Achilles' heel of the standing wave mi- ${ }^{75}$ crofludic filtration devices $^{17}$. Here we use the piezo-tube ${ }^{76}$ end to pass or capture particle collectives reproducing porous filter or chromatographic medium character ${ }^{18} \cdot 77$ COMSOL simulations and tests of proteoglycan accumulation within a monotonic force field are described below.

a) Electronic mail: acs14@cam.ac.uk
The piezo-tube is excited by a radio frequency signal and generates a monotonic pressure gradient at its end. The oscillations of the electric field induce the mechanical vibrations in the wall. This energy transfers axially within the adjoining walls between the piezo-tube and adjacent elastomer coupled glass tubes and radially as an internal pressure wave. Energy along the tube walls is governed by the equations of mechanical displacement field $\mathbf{U}_{S}$ and the electric displacement field $\mathbf{D}_{E l}$ :

$$
\begin{aligned}
& \nabla \cdot \mathbf{T}_{S}=-\rho_{S} \omega^{2} \mathbf{U}_{S} \\
& \nabla \cdot \mathbf{D}_{E l}=0
\end{aligned}
$$

where $\rho_{S}$ is the the mass density of the solid, $\omega$ is the angular frequency of the applied voltage and $\mathbf{T}_{S}$ is the mechanical stress tensor induced within the solid.

In a piezoelectric solid, $\mathbf{T}_{S}$ and $\mathbf{D}_{E l}$ are coupled by the linear piezoelectric constitutive matrix. The stresscharge form of these relations can be expressed as:

$$
\left[\begin{array}{c}
\mathbf{T}_{S} \\
\mathbf{D}_{E l}
\end{array}\right]=\left[\begin{array}{cc}
c_{E} & -e^{T} \\
e & \varepsilon_{S}
\end{array}\right]\left[\begin{array}{c}
\nabla \mathbf{U}_{S} \\
-\nabla V_{S}
\end{array}\right]
$$

where $V_{S}$ is the electric potential field within the solid, $c_{E}$ is the elasticity matrix of the material, $e$ is the electromechanical coupling matrix of the material, and $\varepsilon_{S}$ is the electric permittivity matrix of the material. While in a non-piezoelectric material of the flow tubing, the electromechanical coupling matrix $e$ is absent, so the two energy fields can be expressed as:

$$
\left[\begin{array}{c}
\mathbf{T}_{S} \\
\mathbf{D}_{E l}
\end{array}\right]=\left[\begin{array}{cc}
c_{E} & 0 \\
0 & \varepsilon_{S}
\end{array}\right]\left[\begin{array}{c}
\nabla \mathbf{U}_{S} \\
-\nabla V_{S}
\end{array}\right]
$$

The frequency domain of the acoustic pressure field $p_{L}$ in the liquid can be expressed by Helmholtz equation:

$$
\left(\nabla^{2}+k^{2}\right) p_{L}=0
$$


(a)

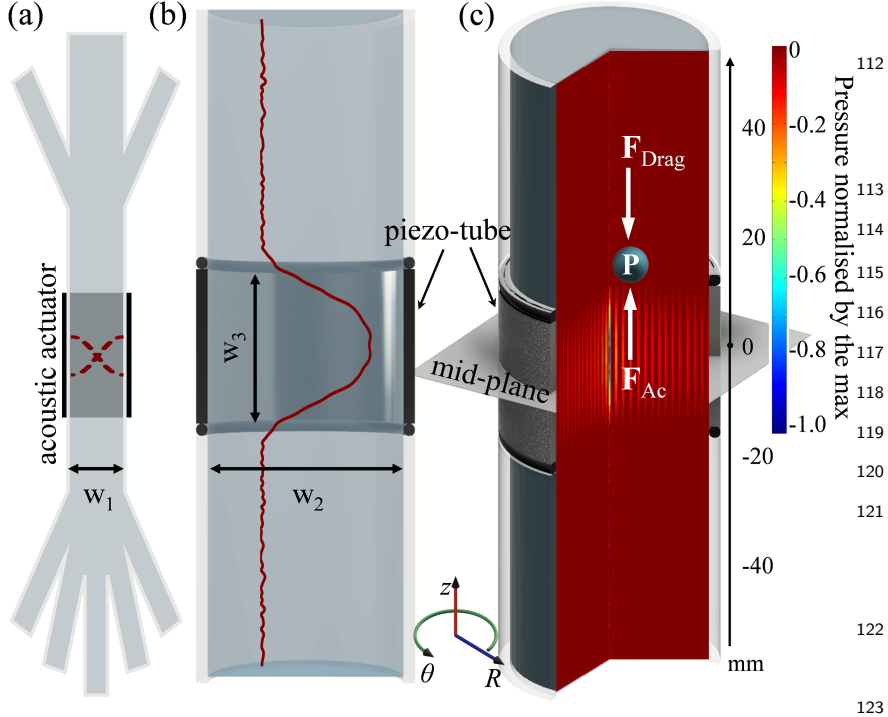

FIG. 1. The pressure gradient profiles in (a) a typical lab-124 on-a-chip acoustic separation setup with multiple inlets and 125 outlets(--- ), where $\mathrm{w}_{1}$ is usually hundreds of microns and (b)126 the reported high-throughput acoustic filter $(-)$, where $\mathrm{w}_{2127}$ and $\mathrm{w}_{3}$ are $34 \mathrm{~mm}$ and $25 \mathrm{~mm}$ respectively. A COMSOL sim- ${ }_{128}$ ulation of the axial acoustic pressure distribution is demonstrated on (c). The acoustic pressure field is symmetric about the mid-plane perpendicular to $z$-axis. It is at its strongest at $z=0$ and decreases along the $z$-axis to both the positive and ${ }^{129}$ negative directions until the pressure approaches a minimum value in liquid within the glass tubes. The tubing geometry ${ }^{130}$ is consisted in the sequence of glass tube, O-ring, piezo-tube, ${ }^{131}$ O-ring and glass tube.
This $z$-axis pressure profile is confirmed by a $\mathrm{COM}_{-135}$ SOL simulation: In FIG.1.(c) an axisymmetric model $\mathrm{in}_{136}$ a cylindrical co-ordinate system is developed. Equations ${ }_{137}$ (1), (2), (3) and (4) are solved simultaneously. The pres- ${ }_{138}$ sure field in the liquid is driven by continuity conditions ${ }_{139}$ of stress and pressure at the tube inner wall. Plane wave ${ }_{140}$ walls of the geometry to model energy leakage outside ${ }_{142}$ the piezo-tube into the flow tubing system.

The particle forces from the non-periodic monotonic ${ }_{144}$ gradient of its ends are determined as follows. If the 145 diameter of the particle, $d_{P}$ is smaller than the acous-146 tic wavelength, the particle is subject to a time aver-147 aged acoustic radiation force $\mathbf{F}_{A c}$, according to Gorkov's148 equation $^{19}$ as: radiation conditions are imposed at the upper and lower ${ }_{141}$
$\mathbf{F}_{A c}=-\frac{\pi}{6} d_{P}{ }^{3}\left(0.5 f_{1} \beta_{L} \nabla<p_{L}^{2}>-0.75 f_{2} \rho_{L} \nabla<v_{L}^{2}>\right)$

where $\beta_{L}$ and $\rho_{L}$ are respectively the isothermal compressibility and the mass density of the liquid, $v_{L}$ is the medium molecular velocity. The coefficients $f_{1}$ and $f_{2}$ can be defined as functions of the liquid and particle compressibilities and densities respectively ${ }^{19,20}$ :

$$
\begin{aligned}
& f_{1}=1-\left(\beta_{P} / \beta_{L}\right) \\
& f_{2}=\frac{2\left[\left(\rho_{P} / \rho_{L}\right)-1\right]}{2\left(\rho_{P} / \rho_{L}\right)+1}
\end{aligned}
$$

where $\beta_{P}$ and $\rho_{P}$ respectively represent the particle compressibility and mass density

Choice of this equation is based on the necessity to calculate the force based on pressure gradients at discrete locations within the tube. This can be contrasted with conventional standing wave forces which are induced in a spatially periodic 1-D sinusoidal field. Under the influence of such a field, Equation (5) can be simplified ${ }^{20}$ to:

$$
\mathbf{F}_{A c}=k V E_{A c} \Phi_{A c} \sin (2 k x)
$$

where $V$ is the particle volume and $E_{A c}$ is the peak energy density dependent on the amplitude of the sinusoidal acoustic pressure field. $\Phi_{A c}$ is the acoustic contrast factor which is a function of the densities and compressibilities of the particle and the liquid (Equation (6)). For a 1-D acoustic field the contrast factor becomes ${ }^{20}$ :

$$
\Phi_{A c}=f_{1}+1.5 f_{2}
$$

Here, the energy density multiplied by a sinusoidal profile gives a force periodicity less than $1 \mathrm{~mm}$. Whereas the piezo-tube, with its non-periodic, monotonic acoustic pressure field as predicted by the COMSOL simulation, generates monotonic gradients separated by the piezotube length matching the form of Equation (5). This gradiant behaviour is consistent with a fraction of acoustic energy leaking out of the ends of the piezo-tube. This profile overlays the underlying radial component of the Bessel field within the fluid volume ${ }^{21}$. This hybrid acoustic force structure supports a particle concentration mode via the tube end geometry as the force determining variable.

The uniqueness of the present work lies in the physics of the tube-end pressure gradient for trapping particles and against a co-linear fluid flow. Whereas the remaining terms involving fluid pressure acting on the particle is based on known physics, i.e. the diameter of the particle $d_{P}$ to the 3rd power, the energy density of the acoustic field $E_{A c}$ and finally the acoustic contrast factor $\Phi_{A c}$. 

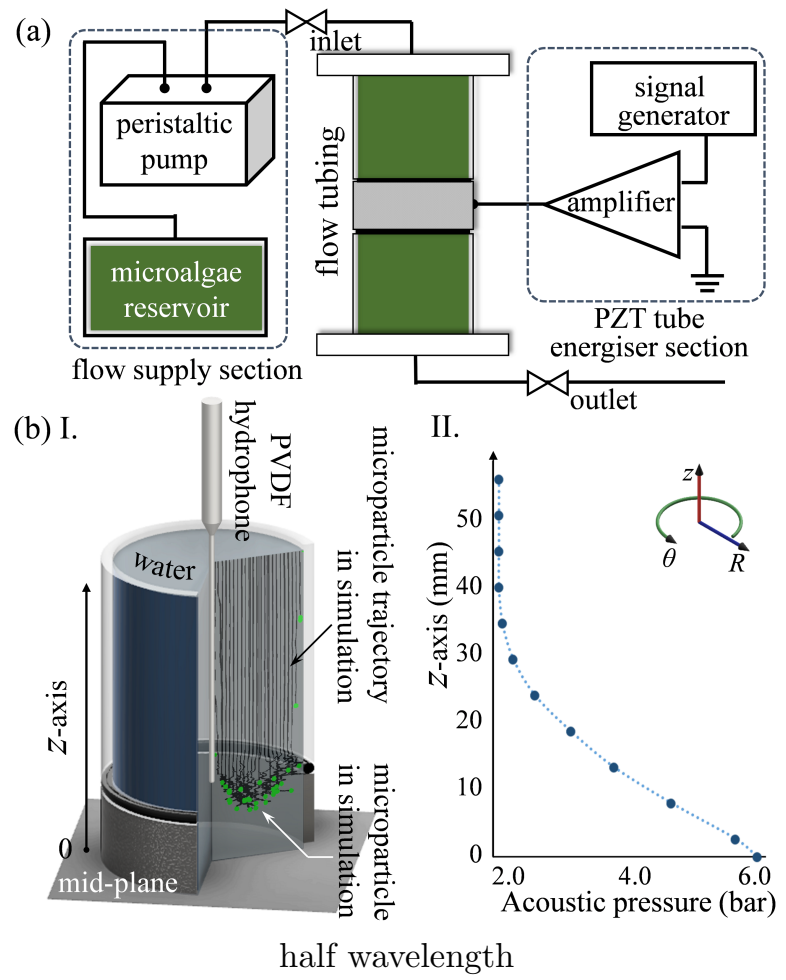

FIG. 2. (a) The high-throughput particle concentration ex- ${ }^{198}$ perimental setup: Acoustic filter configuration based on a flow ${ }^{199}$ tubing system with passive/active tubes, piezo-tube energiser ${ }^{200}$ section and a flow supply system. (b) A COMSOL simulation ${ }^{201}$ predicts particle $(\bullet)$ trajectory $(-)$ in a flow tubing system ${ }^{202}$ coming to a halt near the piezo-tube entrance (I). A PVDF203 hydrophone placed between active and passive tubes helps204 confirm the pressure variations of the barrier plane (II).

Overall transiting particles experience a significant net force field $\mathbf{F}_{\text {Total }}$, which is a vector sum of the acoustic $\mathbf{F}_{\text {Drag }}$ downwards due to the flow rate $\mathbf{u}_{L}$, i.e.:

$$
\begin{aligned}
& \mathbf{F}_{\text {Total }}=\mathbf{F}_{A c}+\mathbf{F}_{\text {Drag }} \\
& \mathbf{F}_{\text {Drag }}=3 \pi \eta_{L} d_{P} \mathbf{u}_{L}
\end{aligned}
$$

where $\eta_{L}$ is the dynamic viscosity of the liquid.

Thus particle paths resulting in concentration or leakage processes, is significantly influenced by the field $\mathbf{F}_{\text {Total }}$ which needs to exceed zero to concentrate particles. For flow rates sufficiently strong or for weak acoustic powers, $\mathbf{F}_{\text {Drag }}$ dominates over $\mathbf{F}_{A c}$ and the particles flow along without being manipulated. As one keeps increasing the particle diameter or power, the acoustic trapping forces increase more strongly (cubic scaling) than the flow-induced drag forces (linear scaling). Hence would have a critical particle diameter below which the acoustic forces are too weak to trap particles against the $e_{171}$ drag forces.

TABLE I. A comparison list of density $\rho_{P}$, compressibility $\beta_{P}{ }^{22}$, acoustic contrast factor $\Phi_{A c}$ and the maximum acoustic force $\left|\mathbf{F}_{A c}\right|_{\max }$ of selected particles. $\left|\mathbf{F}_{A c}\right|_{\max }$ is calculated assuming the particle with a unit size, i.e. $1 \mu \mathrm{m}$, in an acoustic field introduced by a piezo-tube energised with $30 \mathrm{~V}_{p p}$ input at approximately $1.15 \mathrm{MHz} .\left|\mathbf{F}_{A c}\right|_{\max }$ can be scaled as a function of the particle sizes by multiplying $d_{P}^{3}$.

\begin{tabular}{c|c|c|c|c}
\hline particle & $\begin{array}{c}\rho_{P} \\
\mathrm{~kg} / \mathrm{m}^{3}\end{array}$ & $\begin{array}{c}\beta_{P} \\
\mathrm{~Pa}^{-1}\end{array}$ & $\Phi_{A} c$ & $\begin{array}{c}\left|\mathbf{F}_{A c}\right|_{\max }{ }^{\mathrm{a}} \\
\mathrm{pN}\end{array}$ \\
\hline Microphyte $^{22}$ & 1100 & $3.83 \times 10^{-10}$ & 0.243 & 0.088 \\
WBC $^{23} \mathrm{~b}$ & 1090 & $3.59 \times 10^{-10}$ & 0.287 & 0.119 \\
Fused silica $^{24}$ & 2210 & $1.00 \times 10^{-11}$ & 1.648 & 0.577 \\
Polystyrene $^{25}$ & 1050 & $2.49 \times 10^{-10}$ & 0.495 & 0.263 \\
Gold $^{26}$ & 19300 & $5.56 \times 10^{-12}$ & 2.374 & 0.584 \\
\hline
\end{tabular}

a COMSOL predicted numbers

b White blood cell

are loci where the axial trapping force can vanish. These permeable regions are expected to occur along inner cylindrical planes representing the pressure nodes of the field.

Concentration or leakage is also dependent on particle contrast factors when suspended in water as shown in TABLE I. This presents the estimated traction forces in the centre of the tubular geometry according to particle type. This demonstrates dense rigid particles such as gold and fused silica are entrained by significantly higher forces, whilst biological cells have the least effect. Distinguishing from standing waves, it is important to note the axial forces are not periodic along the fluid flow direction. This results in an advantageous single concentration phase.

Particle flow rate, according to Poiseuille/laminar flow (Reynolds number for the flow rate and tube ID used in this work is 12.482), is expected to vary from the centre to the edge. At the centre it is likely to be flowing faster but also working against a stronger field (Bessel function distribution), hence there is a degree of compatibility between the flow rate and force field distribution. Overall choice of different particles of $\beta_{P}, \rho_{P}$ result in different $\Phi_{A c}$, together with $d_{P}$ lead to a variation in the $\mathbf{F}_{A c}$. The larger the $\left|\mathbf{F}_{A c}\right|$ is, the easier it is to manipulate the particle. TABLE. I indicates that biological cells suspended in water are the most difficult particle to capture with acoustic waves, hence for our initial experiments we have selected a biological particle; microphyte as the most challenging test for the system.

An experimental setup for demonstrating the anticipated field-particle coupling of a suspension comprising proteoglycan tracer particles (microphytes $d_{P}$ of $5 \mu \mathrm{m}$ ) is shown in FIG.2.(a). The flow tubing geometry consists of a piezo-tube with an OD $38 \mathrm{~mm}$, ID $34 \mathrm{~mm}$ and height $25 \mathrm{~mm}$ (APC International Ltd., Type I) co-linear with two 40mm glass tubes of the same OD and ID positioned above and below. To minimise acoustic transmis- 
sion an O-ring is inserted between adjoining active and 266 passive tubes. The outer ends of the glass tubes are sand-267 wiched between two customised acrylic sheets with liquid 268 inlet (top) and outlet (bottom) respectively. A sinusoidal ${ }_{269}$ signal from the signal generator (Agilent 33120A Func-270 tion/Arbitrary Waveform Generator) drives the piezo-271 tube at its radial mode resonant frequency and ampli-272 fied by a power amplifier (EIN 310L) to approximately273 $30 \mathrm{~V}_{p p}$. The flow of the particle suspension is driven by 274 a peristaltic pump (Masterflex). The glass tubes allow 275 visualisation of the particle concentration effect.

These demonstration experiments are setup to col-277 lect acoustic and optical data from the tubes via a hy-278 drophone and camera respectively: For measuring the 279 pressure field a PVDF hydrophone (Dr Mueller Instru-280 ments, Müller-Platte Needle Probe) was placed at the 281 tubes axial centre (FIG.2.(b).I.) and the sampled signal ${ }_{282}$ amplified by a voltage amplifier (Dr Mueller Instruments,283 MVA 10). The result is displayed on an oscilloscope 284 (Hantek, DSO5102P) revealing the corresponding plot285 shown in FIG.2.(b).II. and confirming the acoustic pres-286 sure falls significantly outside the piezo-tube.

Under the same conditions we pass the tracer parti-288 cle suspension through the energised piezo-tube to get289 the concentration effect. Of note is the pumps flow rate,290 which is significantly greater than typical lab-on-chip de-291 vice process by 1000 to 10000 times i.e. at $20 \mathrm{~mL} / \mathrm{min} .292$ As the tube shape and depth prevent straightforward293 concentration measurements, the signature chromatic co-294 ordinate $\left(S g_{c c}\right)$ technique was chosen based on its suc-295 cess with assessing green foliage levels from satellite296 images $^{27,28}$ and the potential to profile a smaller photo-29 synthetic system. Here the $S g_{c c}$ carries the RGB colour 298 information of an image correlated with the concentra-299 tion process. A $75 \mathrm{~s}$ video is converted into an image se-300 quence; and a consistent region of interest (ROI) is anal-301 ysed frame by frame. The RGB information is recorded 302 and the $S g_{c c}$ is calculated, ratioing the signature colour 303 digit number over the sum of the red, green and blue 304 digit numbers. The green colour is the most represen-305 tative parameter, as long as the lighting levels remain 306 fixed.

At this stage we observe a concentration effect which ${ }_{308}$ agrees with the form of the theoretical prediction, 309 thus making it a suitable candidate geometry for high-310 throughput solid-liquid separation. A detailed COMSOL 311 simulation also confirms the trajectory of microparticles312 in the flow tubing, which shows the same accumulation ${ }_{313}$ behaviour of the particles at the entrance of the piezo- ${ }_{314}$ tube as expected (FIG.2.(b).I).

Particle concentration and other motional effects were 316 also observed: Particles influenced by the piezo-tube in ${ }_{317}$ either on-state or off-state is presented in FIG.3.(a). This 318 shows the on-state creates a particle concentration ef-319 fect as indicated by the relative concentrations via $\mathrm{ROI}_{320}$ $S g_{c c}$ data collected from a vertically oriented tube with ${ }_{321}$ downward flow. Here frames are taken of four quad-322 rants: above and below an active tube, above and be- -323 low an inactive tube (referred as on_above, on_below, off_above and off_below). Interpreting FIG.3.(b), there is a slight difference between baselines relating to small background differences in the first few frames. For the off-state as time progresses, the green and brown dashed lines indicate matching contrast above and below the tube. Whereas the on-state results in an increasing difference: The solid green line confirms that the particles accumulate above the piezo-tube, i.e. unable to enter the piezo-tube. Around frame 40 a minor leakage is visible, which indicates partial percolation of tracer particles through the field.

Leakage and streaming were minor relative to the trapping effect observed. For the former permeability of the field is associated with particle trajectories along the radial nodal planes, where the axial forces are weak. We hypothesise that the leakage is encouraged when the axial radiation pressure depletes along the nodal planes. As a result, we expect the leakage to be positively correlated to the number of the radial modes, although no tests were done to confirm this. However it was observed that leakage operates uniformly over the width, but is difficult to quantify due to agglomeration effects. Outside of the tube, streaming had minimal contribution to the motion of either entrant or leaked particles. From the gradient of the ROI $S g_{c c}$ the accumulation rate in the active tube beyond frame 70 (where the count is most accurate) is doubled, further confirming the trapping effect.

We believe this is the first demonstration of a particle trajectory arrested by an acoustic barrier derived from a large scale monotonic field. The sample flowing within this tubing system is subject to a dominant localising effect, where it concentrates at a significant volume and flow rate of $20 \mathrm{~mL} / \mathrm{min}$ alongside the supernatant phase. These experiments demonstrate that low contrast bioparticles can be filtered, the most challenging case, which bodes well for entities of higher contrast. A clear focal point is produced at the tube end. We no longer need to depend on the characteristics of the standing wave alone, which can become problematic at small scales and high frequencies as the particle distorts the wavefront and leads to tuning variabilities.

The membrane like filter action is the most prominent characteristic of the profile leading to an accessible accumulation which is relatively straightforward to tap. Batch type fluidic operations can immediately be applied and is amenable for series or parallel operation, with the opportunity to boost very low concentrations.

Applications include plant cell collection, animal cell purification, bioreactor systems, geological separations, agricultural processing and sensing enhancement due to the concentration factor. The approach is especially useful for particles with high acoustic contrast. Here high throughput retrofitted systems benefit from less tuning than standing wave approaches. Thus it can work within micro- or macro-liquid processing systems and support low maintenance, non-clogging and low power applications. These are powerful foundations for processing bi- 
(a)

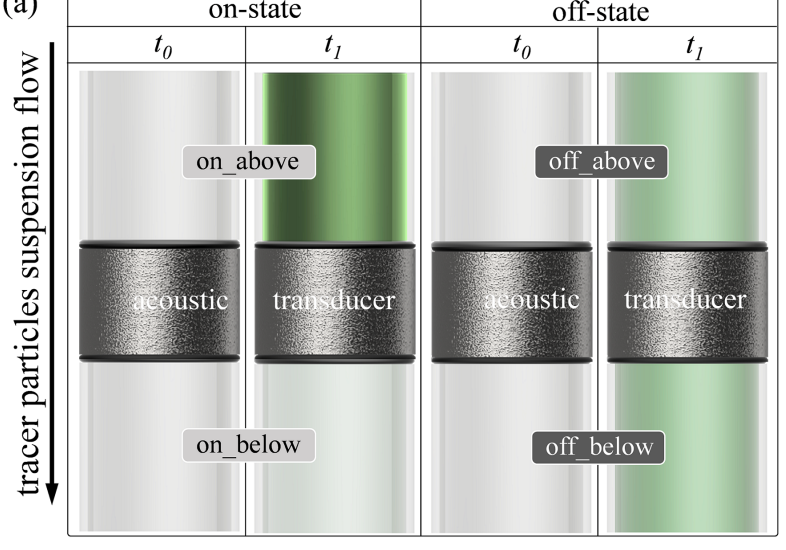

(b)

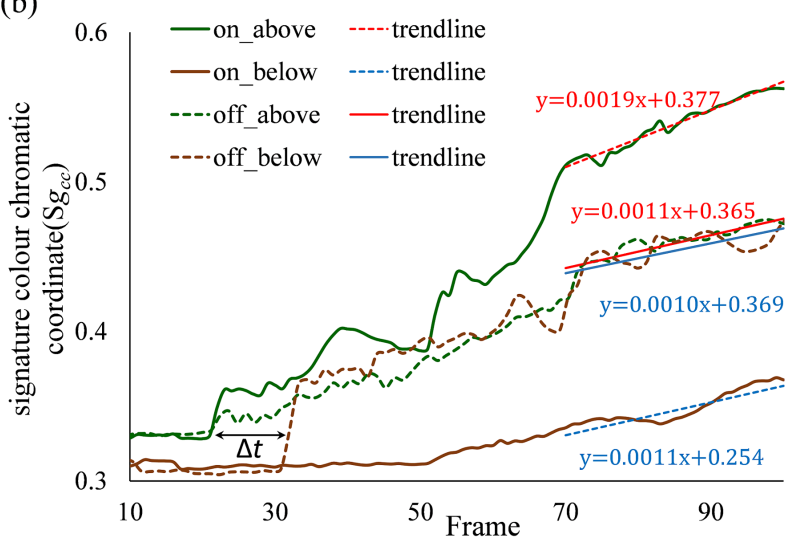

FIG. 3. Visualisation of the filtration action for a tracer particle suspension: Two sets of experiments are utilised, one with the acoustic transducer active (on-state) and the other with it idle (off-state). (a) Images taken at initial $t_{0}$ and endpoint $t_{1}$ (after 60s) show the on-state inhibits particle flow, against a fluid flow of $20 \mathrm{~mL} / \mathrm{min}$. (b) Video frames capture an image sequence for dynamics of the filtration process, with relative concentration obtained from RGB digit numbers of the selected ROI. The number of frames for the tracer particle to transit the acoustic transducer is indicated by $\Delta t$. In the off-state, the marginal difference between the green and brown dashed lines indicates no particle trapping. Whereas, in the on-state, increasing differences between green and brown solid lines indicate a particle concentration effect.

ological molecules including protein and DNA and live 364 cells where high-throughput sorting is available to meet ${ }^{365}$ industrial scale throughput requirements.

The authors gratefully acknowledge funding from the ${ }_{368}^{367}$ BBSRC (BB/NO10310/1) and Innovate UK and thank 369 Dr Devaki Bhatta and Dr William Nock for the helpful 370 discussions.

${ }^{1}$ A. Marzo, A. Ghobrial, L. Cox, M. Caleap, A. Croxford, and ${ }^{374}$ B. Drinkwater, Applied Physics Letters 110, 014102 (2017). 375

${ }^{2}$ L. Cox, A. Croxford, B. Drinkwater, and A. Marzo, Applied ${ }^{376}$ Physics Letters 113, 054101 (2018).

${ }^{3}$ T. Fushimi, T. Hill, A. Marzo, and B. Drinkwater, Applied ${ }^{378}$ Physics Letters 113, 034102 (2018).

${ }^{4}$ H. Li, Y. Wang, M. Ke, S. Peng, F. Liu, C. Qiu, and Z. Liu, ${ }^{380}$ Applied Physics Letters 112, 223501 (2018).

${ }^{5}$ S. Shin, D. Han, M. C. Park, J. Y. Mun, J. Choi, H. Chun, ${ }^{382}$ S. Kim, and J. W. Hong, Scientific reports 7, 9907 (2017). ${ }^{6}$ D. M. Smalley, N. E. Sheman, K. Nelson, and D. Theodorescu, ${ }^{384}$ Journal of proteome research 7, 2088 (2008).

${ }^{7}$ Y.-R. Hu, C. Guo, F. Wang, S.-K. Wang, F. Pan, and C.-Z. Liu, ${ }^{386}$ Chemical Engineering Journal 242, 341 (2014).

${ }^{8}$ A. Patist and D. Bates, Innovative food science \& emerging tech- ${ }^{-38}$ nologies 9, 147 (2008).

${ }^{9}$ H. Bruus, J. Dual, J. Hawkes, M. Hill, T. Laurell, J. Nilsson, ${ }^{390}$ S. Radel, S. Sadhal, and M. Wiklund, Lab on a Chip 11, $3579^{391}$ (2011).

${ }^{10}$ X. Ding, S.-C. S. Lin, B. Kiraly, H. Yue, S. Li, I.-K. Chiang, ${ }^{393}$ J. Shi, S. J. Benkovic, and T. J. Huang, Proceedings of the ${ }^{394}$ National Academy of Sciences 109, 11105 (2012).

${ }^{11}$ P. Augustsson and T. Laurell, Lab on a Chip 12, 1742 (2012). ${ }^{396}$ ${ }^{12}$ B. Jung, K. Fisher, K. D. Ness, K. A. Rose, and R. P. Mariella Jr, ${ }^{397}$ Analytical chemistry 80, 8447 (2008).

${ }^{13}$ F. Trampler, J. M. Piret, S. A. Sonderhoff, and D. G. Kilburn ${ }^{399}$ "Acoustic filter for separating and recycling suspended particles," 400 (1997), US Patent 5,626,767.

${ }^{14}$ M. D. Ward and G. Kaduchak, "Particle analyzing systems and ${ }^{402}$ methods using acoustic radiation pressure," (2014), US Patent ${ }^{403}$ $8,846,408$.

${ }^{15}$ B. Vickroy, K. Lorenz, and W. Kelly, Biotechnology progress 23, 194 (2007).
${ }^{16}$ R. G. Harrison, P. Todd, S. R. Rudge, and D. P. Petrides, Bioseparations science and engineering (Topics in Chemical Engineering, 2015).

${ }^{17}$ P. Dow, K. Kotz, S. Gruszka, J. Holder, and J. Fiering, Lab on a Chip 18, 923 (2018).

${ }^{18}$ T. Imasaka, Analusis 26, 53 (1998)

${ }^{19}$ H. Bruus, Lab Chip 12, 1014 (2012).

${ }^{20}$ C. Reyes, L. Fu, P. P. A. Suthanthiraraj, C. E. Owens, W. Shields IV, G. P. Lopez, P. Charbonneau, and B. J. Wiley, Part. Part. Syst. Charact. 35, 1700470 (2018).

${ }^{21} \mathrm{~J}$. Yang, H. Hwang, Y. M. Bae, M. Kim, and K. Ha, Japanese Journal of Applied Physics 52, 07HE14 (2013).

${ }^{22}$ E. H. Gómez, J. Tryner, A. J. Aligata, J. C. Quinn, and A. J. Marchese, Algal research 31, 77 (2018).

${ }^{23}$ M. Wu, Y. Ouyang, Z. Wang, R. Zhang, P.-H. Huang, C. Chen, H. Li, P. Li, D. Quinn, M. Dao, s. Suresh, Y. Sadovsky, and T. Jun Huang, PNAS 1709210114 (2017).

${ }^{24}$ G. Destgeer, J. Ho Jung, J. Park, H. Ahmed, K. Park, R. Ahmad, and H. Jin Sung, RSC Adv 7, 22524 (2017).

${ }^{25}$ P. Barkholt Muller, R. Barnkob, M. Jakob Herring Jensen, and H. Bruus, Lab Chip 12, 4617 (2012).

${ }^{26}$ P. Kelly, Properties of Materials (CRC Press, 2014).

${ }^{27}$ A. D. Richardson, B. H. Braswell, D. Y. Hollinger, J. P. Jenkins, and S. V. Ollinger, Ecological Applications 19, 1417 (2009).

${ }^{28}$ O. Sonnentag, K. Hufkens, C. Teshera-Sterne, A. M. Young, M. Friedl, B. H. Braswell, T. Milliman, J. OKeefe, and A. D. Richardson, Agricultural and Forest Meteorology 152, 159 (2012).

${ }^{29}$ R. D. Perlack, Biomass as feedstock for a bioenergy and bioproducts industry: the technical feasibility of a billion-ton annual supply (Oak Ridge National Laboratory, 2005).

${ }^{30}$ P. Li, Z. Mao, Z. Peng, L. Zhou, Y. Chen, P.-H. Huang, C. I. Truica, J. J. Drabick, W. S. El-Deiry, M. Dao, et al., Proceedings of the National Academy of Sciences 112, 4970 (2015).

${ }^{31}$ R. Guldiken, M. C. Jo, N. D. Gallant, U. Demirci, and J. Zhe, Sensors 12, 905 (2012).

${ }^{32}$ L. A. Ostrovsky, A. Priev, V. Ponomarev, and Y. Barenholz, in Proceedings of Meetings on Acoustics 162ASA, Vol. 14 (ASA, 2011) p. 020002.

${ }^{33} \mathrm{P}$. Collas and M. Barmatz, The Journal of the Acoustical Society of America 81, 1327 (1987). 